\title{
Synthesis of 2-Methoxybenzoylhydrazone and Evaluation of their Antileishmanial Activity
}

\author{
Mohd Syukri Baharudin ${ }^{1}$, Muhammad Taha ${ }^{1, *}$, Nor Hadiani Ismail ${ }^{2}$, Khalid Mohammed Khan ${ }^{3}$, \\ Faridahanim Mohd Jaafar ${ }^{2}$, Samreen ${ }^{3}$, Salman Siddiqui ${ }^{3}$ and M. Iqbal Choudhary ${ }^{3}$ \\ ${ }^{a}$ Atta-ur-Rahman Institute for Natural Product Discovery, Universiti Teknologi MARA (UiTM), Puncak Alam Campus, \\ 42300 Bandar Puncak Alam, Selangor, Malaysia; ${ }^{b}$ Faculty of Applied Science UiTM, 40450 Shah Alam, Selangor, \\ Malaysia; H.E.J. Research Institute of Chemistry, International Center for Chemical and Biological Sciences, University of \\ Karachi, Karachi-75270,Pakistan;_E-mail:taha_hej@yahoo.com; muhamm9000@puncakalam.uitm.edu.my
}

2-Methoxybenzoylhydrazones 1-25 were synthesized from 2-methoxybenzoylhydrazide which was obtained from methyl-2methoxybenzoate by refluxing with hydrazine hydrate for $5 \mathrm{~h}$ which was then crystallized from methanol. 2Methoxybenzoylhydrazones were prepared by condensing 2-methoxybenzoylhydrazide with different aromatic aldehydes in refluxing ethanol for 3 to 4 hour in high yield. Compounds 1-25 showed varying degrees of antileishmanial activities with $\mathrm{IC}_{50}$ values ranging between $1.95-88 \mu \mathrm{M}$, as compared to standard pentamidine $\left(\mathrm{IC}_{50}=5.09 \mu \mathrm{M}\right)$. Compounds 10 (IC $50=1.95$ $\mu \mathrm{M}), 11\left(\mathrm{IC}_{50}=2.49 \mu \mathrm{M}\right)$, and $2\left(\mathrm{IC}_{50}=3.29 \mu \mathrm{M}\right)$ were found to be more active than standard pentamidine $\left(\mathrm{IC}_{50}=5.09 \mu \mathrm{M}\right)$. Compounds $7\left(\mathrm{IC}_{50}=7.64 \mu \mathrm{M}\right), \mathbf{8}\left(\mathrm{IC}_{50}=13.17 \mu \mathrm{M}\right), \mathbf{1 8}\left(\mathrm{IC}_{50}=13.15 \mu \mathrm{M}\right)$, and $\mathbf{2 4}\left(\mathrm{IC}_{50}=15.65 \mu \mathrm{M}\right)$ exhibited good activities. Compounds $3\left(\mathrm{IC}_{50}=28.24 \mu \mathrm{M}\right), 1\left(\mathrm{IC}_{50}=31.47 \mu \mathrm{M}\right), 12,\left(\mathrm{IC}_{50}=31.56 \mu \mathrm{M}\right), 4\left(\mathrm{IC}_{50}=33.2 \mu \mathrm{M}\right), 15\left(\mathrm{IC}_{50}=34.85 \pm 0.48\right.$ $\mu \mathrm{M}), 5\left(\mathrm{IC}_{50}=35.41 \mu \mathrm{M}\right), 9\left(\mathrm{IC}_{50}=40.07 \mu \mathrm{M}\right)$, and $19\left(\mathrm{IC}_{50}=45.67 \mu \mathrm{M}\right)$ were found to be moderately active. Compounds 13 , 14, 16, 17, 20-23 and 25 showed weak activities with $\mathrm{IC}_{50}$ values between 57.41 to $88.56 \mu \mathrm{M}$. Only compound 6 was found to be completely inactive.

Keywords: 2-Methoxybenzoylhydrazone, antileishmanial activity, pentamidine, leishmania promestigotes. 